\title{
Mesenchymal chondrosarcoma arising in fibrous dysplasia of the femur
}

\author{
J B Blackwell
}

\begin{abstract}
The occurrence of mesenchymal chondrosarcoma in an area of fibrous dysplasia in the upper femur of a 28 year old man is reported. It is believed that this is the first documented example of such an association. A further unusual feature is the presence of benign giant cells in the mesenchymal chondrosarcomatous component.
\end{abstract}

$(\Im$ Clin Pathol 1993;46:961-962)

Mesenchymal chondrosarcoma is rare malignant tumour of either bone or soft tissue with a peak incidence in the second and third decades.

Fibrous dysplasia is a common disorder of bone which is monostotic in $80 \%$ of patients and has a peak age for diagnosis in the first two decades.

Malignant transformation in fibrous dysplasia is a rare but well recognised complication, with osteosarcoma, fibrosarcoma, and chondrosarcoma the tumours most commonly found in that order of frequency.

It is believed that this is the first report of mesenchymal chondrosarcoma in association with fibrous dysplasia.

\section{Case report}

A 28 year old Afghan man presented to a refugee hospital in Pakistan because he was unable to walk. He gave a history of having had increasing difficulty in walking over several years.

Biopsy specimens were taken from the upper and lower components of the lesion and disarticulation planned. A visiting surgeon brought the specimens to Australia as no histopathological facilities were available on site.

\section{Radiological findings}

There was a pathological fracture of the right neck of femur (fig 1). Almost complete destruction of the bony trabeculae in the upper femur and intertrochanteric region was seen and this destructive process extended into the head of femur where only a thin rim of cortical bone remained. On the lateral aspect of the upper femur the cortex had been breached and there was some soft tissue extension of tumour.
The process extended into the upper femoral shaft where a thin zone of reactive sclerosis was seen around the inferior margin. A ground glass appearance was noted, but this may have been, at least in part, due to film type and suboptimal processing. The superior margin of the tumour was less well defined and there was permeated bone destruction extending into the base of the greater trochanter.

The margin of the acetabular fossa was thin and the patient had probably not been weightbearing for a considerable time.

\section{Pathology}

In the inferior part of the lesion there were changes typical of fibrous dysplasia, with multiple small islands of woven bone in a background of benign fibrous tissue (fig 2). The biopsy specimen from the upper part of the lesion showed highly cellular tissue composed of cells with ovoid or occasionally elongated nuclei and numerous mitoses. Isolated cell necrosis was present. Plentiful benign

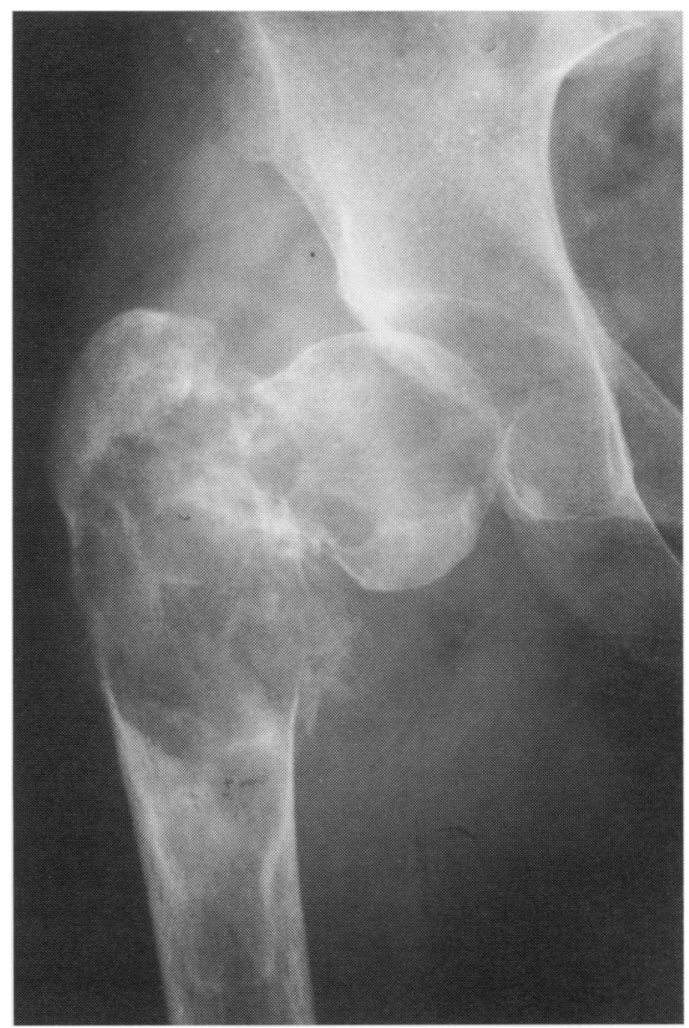

Figure 1 Destructive and lytic lesion in upper right femur with pathological fracture. In lower third margin is well demarcated.

\section{Hospital, 175 \\ Cambridge St Subiaco, Wester J B Blackwell \\ Correspondence to: J B Blackwell


Figure 2 Area of fibrous dysplasia with small islands of bone in moderately cellular fibrous tissue.

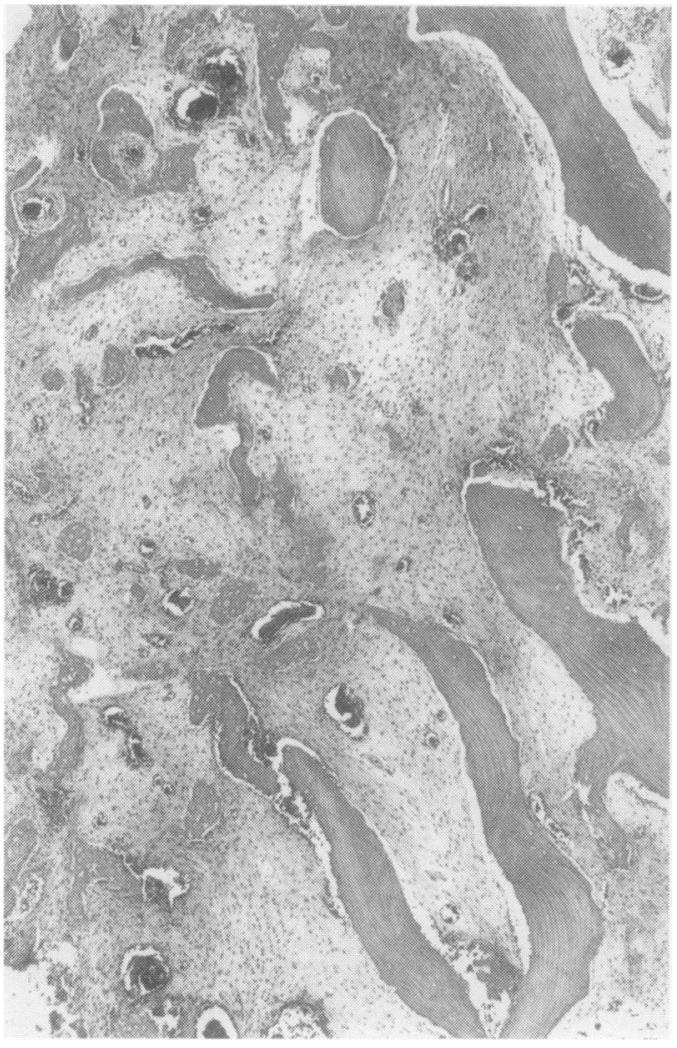

multinucleated giant cells were scattered throughout the lesion (fig 3) and some of these lined large vascular spaces. Scattered throughout the lesional tissue were small islands of fairly mature cartilage which was devoid of nuclear atypia (fig 4).

Mesenchymal chondrosarcoma arising in fibrous dysplasia was diagnosed.

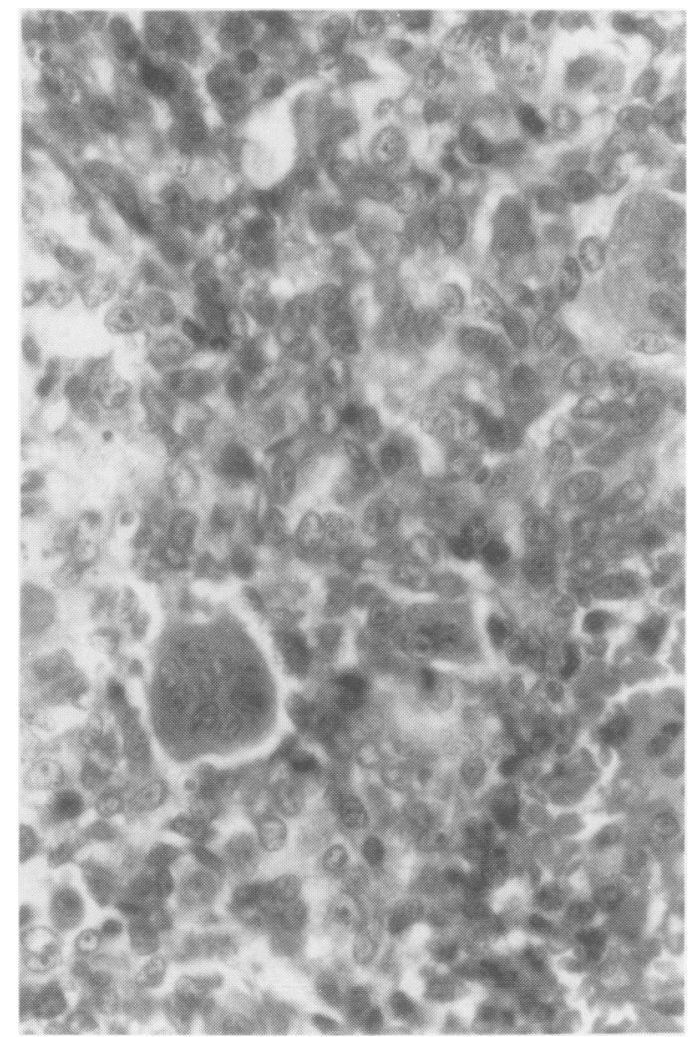

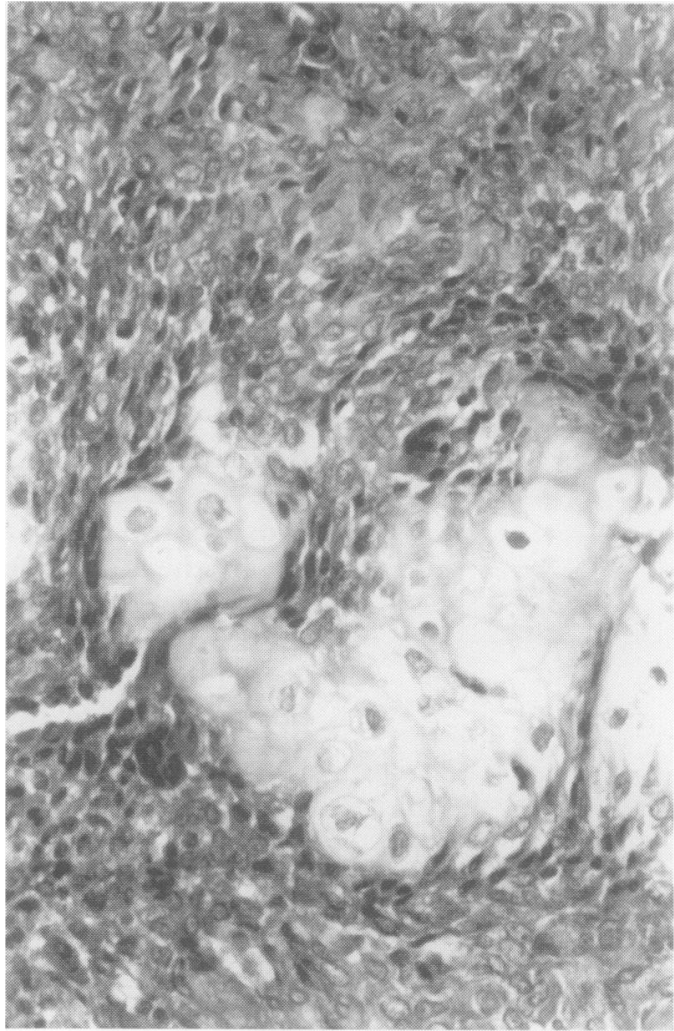

Figure 4 Cartilage island in spindle cell tumour.

\section{Discussion}

The histological features in this case were not those of a classic mesenchymal chondrosarcoma, but after careful consideration this was the preferred diagnosis of four pathologists experienced in bone tumour pathology.

In the differential diagnosis chondroblastoma with secondary aneurysmal bone cyst was considered because of the presence of cartilage and the benign multinucleated giant cells, but the stromal cells were not typical and there was excessive cellularity and a high mitotic rate. This diagnosis did not correlate with the radiological findings.

Benign multinucleated giant cells are not a recognised feature of mesenchymal chondrosarcoma but they have been described.'

Malignant transformation in fibrous dysplasia is a rare but well recognised complication. In a review of the published findings in 1988, Yabut et $a l^{2}$ found 83 such cases.

Osteosarcoma, with 40 cases, was the most common, followed by fibrosarcoma $(n=22)$, and chondrosarcoma $(n=11)$. No case of mesenchymal chondrosarcoma was documented. Patients developing sarcoma were usually in the third or fourth decade and the common sites were craniofacial, femur, and tibia.

I thank Mr T Keenan for making available the surgical specimen, Dr I Morrison for radiological interpretation, and Dr AJ Malcolm for review of the pathology. Ms L West typed the manuscript.

1 Scheithauer BW, Rubinstein LJ. Meningeal mesenchymal chondrosarcoma. Cancer 1978;42:2744-52.

2 Yabut SM Jnr, Kenan S, Sissons HA, Lewis MM. Malignant transformation of fibrous dysplasia. A case report and review of the literature. Clin Orthop 1988; 228:281-9. 